\title{
TV/Series
}

$2 \mid 2012$

Les séries télévisées dans le monde : Échanges, déplacements et transpositions

\section{Préface. Les séries télévisées dans le monde : Échanges, déplacements et transpositions}

Sylvaine Bataille et Sarah Hatchuel

\section{(2) OpenEdition}

\section{Journals}

Édition électronique

URL : http://journals.openedition.org/tvseries/1351

DOI : 10.4000/tvseries. 1351

ISSN : 2266-0909

Éditeur

GRIC - Groupe de recherche Identités et Cultures

Référence électronique

Sylvaine Bataille et Sarah Hatchuel, «Préface. Les séries télévisées dans le monde : Échanges, déplacements et transpositions », TV/Series [En ligne], 2 | 2012, mis en ligne le 01 novembre 2012, consulté le 24 septembre 2020. URL : http://journals.openedition.org/tvseries/1351 ; DOI : https:// doi.org/10.4000/tvseries.1351

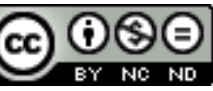

$T V /$ Series est mis à disposition selon les termes de la licence Creative Commons Attribution - Pas d'Utilisation Commerciale - Pas de Modification 4.0 International. 


\section{Préface}

Les séries télévisées dans le monde : Échanges, déplacements et transpositions

Sylvaine BATAILlE et Sarah HATCHUEL

$\mathrm{P}$ résentes dans le paysage télévisuel à l'échelle nationale comme à l'échelle mondiale, les séries de fiction sont des produits culturels transnationaux, qui circulent entre les pays et traversent les frontières. Envisager les séries télévisées comme un objet d'étude international, les placer dans le monde, suscite des questionnements sur les enjeux géopolitiques, idéologiques et esthétiques que recouvrent leurs déplacements ainsi que les transpositions et les échanges culturels accompagnant cette mobilité. Il s'agit également de « spatialiser » la fiction sérielle : la mettre en lien avec l'espace où elle est créée ou diffusée, ou encore s'intéresser aux représentations spatiales et géographiques qu'elle construit.

\section{Échanges : « Mirror, Mirror »}

La relation que la série entretient avec son contexte de production est spéculaire : le reflet plus ou moins déformé que renvoie la fiction à la société qui l'a produite est susceptible, par les réactions suscitées chez les téléspectateurs, de contribuer à former ou à modifier leur appréhension du monde; cela est d'autant plus prégnant dans le cas des séries télévisées, du fait de leur récurrence même et des phénomènes d'engouement qu'elles provoquent.

Dans le monde créé par la fiction, certains lieux du territoire d'origine de la série, des lieux familiers ou emblématiques, représentés, dans leur dynamique, avec plus ou moins d'exactitude, se trouvent offerts à la discussion. Plus largement, dans le miroir tendu par la série, une société se regarde et se réfléchit, pour construire ou affirmer une/des identité(s) et porter un discours critique sur ses valeurs fondatrices ou son mode de vie, notamment par le biais de représentations utopiques ou dystopiques. Dans l'article coordonné par Arnaud Brennetot et Gérald Billard sur le mythe de la banlieue résidentielle dans les séries américaines, les auteurs dévoilent comment l'utopie que représentait autrefois la suburb (en réconciliant la ville moderne et la campagne, et en permettant l'exercice d'une vie libre dans le cadre de petites communautés) s'est progressivement transformée en dystopie angoissante. Des séries comme Dexter (Showtime, 2006-), Weeds (Showtime, 2005-) ou Desperate Housewives ( $\mathrm{ABC}$, 2004-2012) remettent en question un modèle suburbain qui ne protègerait pas de la monstruosité, de la déviance ou

\footnotetext{
${ }^{1}$ Titre d’un épisode de Star Trek (NBC, 1966-69).
} 
de la démoralisation, et formulent, ce faisant, une véritable critique socio-politique de l'idéal américain.

Cet idéal américain est également mis à mal, de manière certes plus indirecte, par la série Star Trek (NBC, 1966-69). Le titre d'un de ses épisodes, « Mirror, Mirror » (2.4) semble donner une clé de lecture de la série elle-même. L'article de Donna Andréolle établit ainsi un parallèle entre les pérégrinations spatiales du vaisseau Entreprise et le mythe américain de la Frontière, la quête incessante de nouvelles terres à conquérir afin de perpétuer le projet utopique d'une société démocratique. Cependant, les planètes découvertes par l'équipage du Capitaine Kirk ne sont pas aussi paradisiaques que prévu : l'utopie s'avère ambiguë, reflétant la remise en question du rêve américain dans le contexte de la contreculture des années 6o. La série Star Trek peut ainsi être vue comme un site culturel médian où se négocie à la fois la contestation du passé et la réinvention de l'avenir.

Au contraire, la Catalogne devient, dans la série Ventdelplà (TV3, 2005-2010), « un monde politiquement, culturellement et linguistiquement idéal ». Comme le souligne Jennifer Houdiard, même si la série s'inscrit dans un espace géographiquement identifiable et entend présenter la société catalane et sa ruralité de manière réaliste, elle construit finalement une utopie peu vraisemblable où l'identité de la Catalogne n'est jamais remise en question, à l'image du village créé spécifiquement pour la fiction.

Entre fiction et réalité, à fois espace de dystopie et de séduction, le Village de la série Le Prisonnier (The Prisoner, ITV, 1967-68) joue de son indétermination toponymique et géographique. Valérie Foulquier explore ses méandres sous la double modalité de la perspective et du déplacement. Le Village entraine les spectateurs dans un univers ludique et labyrinthique où le lieu est mis en scène et où l'espace organisé se délite pour nous faire entrer dans un univers onirique aux accents surréalistes. La critique sociale que représente cette prison dorée est adaptée dans une deuxième version du Prisonnier, diffusée en 2009 sur AMC. Comme l'analyse Sébastien Lefait, la nouvelle dystopie prend en compte l'importance accrue de la surveillance dans nos sociétés contemporaines : la série révèle alors une mise en abyme vertigineuse où la vie « réelle » surveillée est « fictionnalisée ».

Dans The West Wing (NBC, 1999-2006), ainsi que le montre Eric Gatefin, les États-Unis sont également «fictionnalisés » et apparaissent généralement sous la forme d'une carte des États ou sur un écran de télévision : l'image du pays est ainsi reconstruite dans les dialogues et les réactions de l'équipe présidentielle, au sein d'un microcosme étriqué fait de salles vitrées et de couloirs, qui vient nier les réalités sociales du pays.

À travers le prisme de la fiction, un pays peut s'interroger sur ses relations avec un voisin, une puissance rivale ou une ancienne colonie. Les séries télévisées peuvent s'ancrer fortement dans des 
problématiques propres à l'environnement où elles sont produites, en agissant comme le révélateur de certaines peurs, fantasmes ou préoccupations nationales et en entrant souvent en interaction avec les autres représentations dont disposent les téléspectateurs, parfois dans un but ouvertement didactique ou politique (par exemple, renforcer la cohésion sociale), parfois de façon beaucoup plus ambigüe. La série britannique The Jewel in the Crown (ITV, 1984), située en Inde au moment de l'indépendance et de la partition, est analysée par Florence Cabaret comme un feuilleton qui, tout en reflétant les changements économiques et sociaux de la Grande-Bretagne dans les années 80, aborde le passé colonial dans une tension permanente entre un conservatisme nostalgique, où l'ancienne colonie est toujours vue à travers un regard avant tout britannique, et une réévaluation relative de l'histoire, où l'idéologie dominante est révélée comme telle, mais de manière encore furtive. L'article d'Amandine Ducray sur les hybridations et oscillations identitaires dans les sitcoms ethniques anglaises montre à quel point ces séries (ainsi que leur évolution depuis les années 70) viennent refléter et susciter sur le long terme des questionnements complexes en matière de relations raciales en Grande-Bretagne.

\section{Déplacements : circulation et adaptation}

L'ancrage national des séries télévisées n'est pas nécessairement un obstacle à leur réception dans des pays étrangers, si bien que de nombreuses séries articulent une dimension locale avec une circulation globale. Certaines questions socio-culturelles soulevées dans une série à propos de son pays de production sont à même de mobiliser les spectateurs d'autres pays, notamment lorsque ceux-ci sont voisins ou ont des contacts réguliers, ou bien lorsqu'ils partagent des références culturelles, lorsqu'une histoire commune les lie, que ce lien soit amical ou qu'au contraire il soit marqué par la rivalité et le conflit. On se souvient de la réception de Dallas (CBS, 1978-91) en Union soviétique et dans ses pays satellites dans les années quatre-vingt. Diffusée parce qu'elle avait été perçue comme une preuve des vices de l'impérialisme et du capitalisme américains, la série a peut-être, en fait, contribué à l'effondrement du bloc de l'Est en affichant les richesses occidentales ${ }^{2}$.

La proximité géographique ne s'avère pas constituer une condition indispensable au succès de l'exportation : par certains décors et personnages, ou par l'évocation d'expériences qui se trouvent partagées, une série peut plaire à un public étranger résidant dans un pays éloigné du lieu de production en termes de distance

${ }^{2}$ Voir Nick Gillespie et Matt Welch, « How Dallas Won the Cold War », The Washington Post, 27 avril 2008, http://www.washingtonpost.com/wpdyn/content/article/2008/04/25/AR2008042503103.html, consulté en août 2012. 
topographique, mais finalement proche du fait de ses préoccupations, de son environnement et de ses modes de vie. La circulation à l'échelle planétaire de normes en matière d'habitation, d'architecture, de déplacement, ou encore d'habillement et d'équipement rendent bon nombre de discours aisément transférables. Dans son article sur la créolisation des telenovelas, Eliane Wolf explique le succès de productions sud-américaines à l'T̂le de La Réunion par une «proximité historique et socioculturelle » entre le lieu de création originel et celui de diffusion, à travers l'expérience de la colonisation et de l'oppression, le métissage, le système de "plantation », et l'hybridité des modes de vie oscillant entre tradition et modernité. Eliane Wolf montre notamment que les tentatives d'adaptations locales (comme le doublage en créole ou la copie produite localement) ne supplantent pas, bien au contraire, les telenovelas originales.

Il n'en reste pas moins que l'enracinement de la fiction dans le contexte local de production peut parfois être considéré, à différentes étapes du processus allant de la création à la diffusion, comme un handicap mettant en danger la rentabilité de son exportation. Certaines séries, par leur traitement des lieux, semblent, dans leur conception même, immunisées d'office contre les risques que pourrait leur faire courir une identité nationale marquée: la telenovela se fonde ainsi traditionnellement sur des intrigues sentimentales ${ }^{3}$ qui se déroulent dans un cadre conçu sur le mode de l'évasion et du rêve 4 ; le soap opera se caractérise par une "absence ou atrophie de l'espace sensible », « [s]ans doute parce que le lieu réel du soap est ailleurs, au sein de la télévision elle-même 5 ».

Des stratégies d'adaptation sont également développées pour prémunir le produit culturel de l'échec à l'exportation : la circulation d'une série s'accompagne de mutations plus ou moins poussées, si bien qu'elle apparaît comme un objet évolutif, qui reste rarement figé dans une forme originelle. Elle forme ainsi un terrain particulièrement propice aux entreprises d'acclimatation, de domestication et d'appropriation. Ces interventions sur la série originale vont de l'ajout de sous-titres (intervention minimale), à la réécriture complète

\footnotetext{
3 Une définition souvent citée du modèle de la telenovela est la suivante : « c'est l'histoire d'un couple qui veut s'embrasser mais que le scénariste a décidé, sur près de 200 épisodes, de ne pas laisser faire » (voir Frédéric Martel, Mainstream, Enquête sur cette culture qui plaît à tout le monde, Flammarion, 2010, p. 285).

4 Selon l'actrice de telenovela Cynthia Olavarria, « ce sont des histoires qui donnent de l'espoir, qui emmènent le téléspectateur dans un monde de rêve. Et devant une telenovela, on oublie tout. » (propos cités dans « La 'telenovela' se réinvente pour le public latino aux États-Unis ", L'Express. $f r$, article publié le 31 août 2011, consulté en octobre 2012 http://www.lexpress.fr/actualites/1/economie/la-telenovela-se-reinvente-pour-le-publiclatino-aux-etats-unis 1025732.html)

5 Jean Mottet, "L’espace-temps de la télévision : le cas du soap opera », Quaderni, n9, 1989-1990, p. 76 [p. 65-78].
} 
(intervention maximale), en passant par le doublage ou la standardisation linguistique, par exemple.

Deux articles se concentrent sur l'adaptation d'une série dans une même langue : de l'anglais à l'anglais dans le cas de The Office et du français au français dans le cas des Invincibles. Se pose alors la question des raisons mêmes de l'adaptation. Shannon Wells-Lassagne explore les ressorts de la transformation du The Office britannique (BBC, 2001-2003) pour l'accorder aux goûts américains (NBC, 2005-), alors que, paradoxalement, les références culturelles de la version anglaise étaient déjà fortement américaines ou, au moins, " internationales ». La logique de l'adaptation est à rechercher, en fait, dans les tensions génériques et idéologiques que présente la série originelle. L'épisode-pilote américain atténue les situations dérangeantes et insiste sur les caractéristiques de la sitcom, réduisant ainsi l'ambiguïté liée au genre, entre «documentaire », « mocumentaire » et « fiction ».

Sathya Rao et Justine Huet montrent, à leur tour, que l'adaptation française (Arte, 2010-) de la série québécoise Les Invincibles (Radio-Canada, 2005-2009) a consisté tout autant à refléter la réalité culturelle et nationale du public cible en jouant, par exemple, sur les horaires de programmation, sur le casting, la chanson du générique et les références intertextuelles, qu'à opérer des choix stylistiques spécifiques en termes de temps (durée et rythme des épisodes) et d'espace (choix des lieux, échelle des plans, profondeur de champ, chromatisme). Cependant, en retenant une part de l'étrangeté originelle, l'adaptation de la série en France a peut-être permis de renouveler l'imaginaire de l'espace télévisuel d'accueil.

Ces efforts d'adaptation ne sont pas systématiquement couronnés de succès. Les échecs que rencontrent parfois, auprès des publics visés, les entreprises de remaniement et de réécriture soulèvent certaines questions : les téléspectateurs n'apprécient-ils pas davantage l'étrangéité des séries étrangères que ne voudraient le croire les diffuseurs (en témoigne par exemple l'engouement des Anglais pour la série de Canal +, Engrenages, diffusée outre-Manche par BBC Four sous le titre Spiral). La volonté des diffuseurs de familiariser l'étranger n'est-elle pas le signe d'une frilosité ? Ne contribue-t-elle pas à aplanir les particularités de la série pour en faire un produit formaté qui plaira à coup sûr, du moins le pense-t-on? À l'inverse, la variété des adaptations n'est-elle pas le signe que, malgré la circulation mondiale des images, le monde conserve une hétérogénéité culturelle salutaire ? La nécessité de l'adaptation montrerait alors que de nombreuses séries sont écrites pour des publics avant tout locaux : leur contenu ne ferait pas, dès les premiers stades de la production, l'objet d'une homogénéisation qui viserait, littéralement, à "plaire à tout le monde ». La création, la diffusion et la réception des séries témoignent 
ainsi d'une tension entre le «local» et le «global», tension qui se reflète dans les néologismes « glocal » et « globalocal ».

La circulation des séries dans le monde implique nécessairement des relations géopolitiques. Dans son ouvrage Mainstream, Enquête sur cette culture qui plaît à tout le monde, Frédéric Martel explore « la géopolitique de la culture et des médias à travers le monde ${ }^{6}$ », et inclut notamment un chapitre sur les feuilletons télévisés7. Les séries dites " américaines », c'est-à-dire en provenance des États-Unis, sont supposées « envahir » les programmes des chaînes des autres pays. On pourrait alors interpréter la prédominance des séries américaines selon un modèle centre-périphérie dans lequel les États-Unis domineraient le reste du monde culturellement, en exerçant le «soft power » cher à Joseph $\mathrm{Nye}^{8}$, forme de pouvoir fondée non pas sur la force ou sur la coercition, mais sur la séduction des publics et la propagation indirecte - que d'aucuns pourraient qualifier d'insidieuse - des «valeurs » et idéologies américaines par le biais de produits culturels exportés massivement. Les effets incontrôlés de la diffusion de la série Dallas en Union Soviétique (voir plus haut) fournissent une illustration de ce « soft power » américain.

Selon ce modèle, l'engouement pour les séries américaines serait le résultat d'une manipulation subtile, totalement indolore, agréable même, d'un piège parfait parce que ceux qui sont piégés n'ont pas (ou peu...) conscience de l'être. Commenter les séries américaines comme nous le faisons, leur donner le statut d'objet d'étude académique reviendrait peut-être, de fait, à les légitimer et à renforcer l'hégémonie des productions américaines sur la culture de masse mondiale.

Cependant un tel modèle se heurte à plusieurs écueils, à commencer par le fait que si message culturel il y a, celui-ci sera reçu et interprété de façons diverses selon les publics. Par ailleurs, la série télévisée n'est pas un canal déversant un message univoque dans le cerveau des téléspectateurs, mais un site de négociation culturelle où s'affrontent des idéologies et des points de vue différents. L'article d'Eliane Wolff sur la réception des telenovelas montre qu'il est difficile de prévoir ce que le public va apprécier, et combien l'étude sociologique de la réception est complexe et requiert une méthodologie rigoureuse, adaptée au contexte.

De nombreuses séries américaines suscitent, de la part de leurs spectateurs, des interprétations variées, divergentes, voire des contresens. En outre, le modèle du téléspectateur passif s'écroule à mesure

\footnotetext{
${ }^{6}$ Martel, p. 12.

7 Voir Martel, p. 269-297 : chapitre 12, « Géopolitique des dramas, feuilletons du ramadan et autres telenovelas $»$

${ }^{8}$ Joseph Nye, Bound to Lead: The Changing Nature of American Power, New York, Basic Books, 1990; et Soft Power: The Means to Success in World Politics, New York, Public Affairs, 2004.
} 
que se développent les blogs, forums et autres plateformes d'expression libre et individuelle sur Internet.

D'autres facteurs, plus géographiques, remettent également ce modèle en question. Les États-Unis se trouvent, en effet, " courtcircuités " dans un certain nombre de flux d'importation et d'exportation : dans Mainstream, Martel montre ainsi que les ÉtatsUnis «ne sont plus les seuls à manier le soft power, à produire des contenus et à les exporter ${ }^{9}$ » et ne sont donc plus l'unique centre d'influence. L'Amérique du Sud et la Corée du Sud sont des centres émergents d'où se diffusent des contenus culturels au reste du monde, comme l'a par exemple développé Erika Thomas dans son ouvrage Les Telenovelas entre fiction et réalité (L'Harmattan, 2003). Dans son article sur la " Vague Coréenne », Stéphane Thévenet analyse d'ailleurs le rapport entre les productions coréennes et le reste du monde à l'intérieur de la diégèse même des séries. À partir de la fin des années 1990 et jusqu'en 2006 (où commence alors une crise de la production), les téléspectateurs coréens assistent, en effet, à l'apparition de séquences, importantes sur le plan narratif, se déroulant à l'étranger. Il s'agit de répondre à une demande d'exotisme et de romantisme, de mettre en scène la réussite des entreprises et des résidents coréens dans d'autres pays et de pouvoir situer l'intrigue dans un espace de liberté et, donc, de transgression, libéré du poids des codes traditionnels.

L'émergence de certains pays du Sud sur le terrain économique se double d'une émergence sur le terrain culturel. Faut-il donc s'attendre à une sorte de guerre des cultures au terme de laquelle un pays prendra la place des États-Unis dans le rôle de la puissance culturelle hégémonique ? À nouveau, cela semble peu en accord avec le rôle actif des publics actuels. On peut penser qu'au contraire le succès rencontré par certaines séries allochtones aura pour effet de stimuler la création télévisuelle autochtone, comme ce fut le cas en France, où le feuilleton Plus belle la vie (France 3, 2004-) est le résultat d'un métissage de formes esthétiques en provenance de l'étranger, comme l'étudie Marine Legagneur. Marie Tréfousse montre, de plus, que Plus belle la vie a également exploité le succès de la série américaine 24 à des fins ludiques : en créant le personnage du policier Jean-Paul Boher en écho à Jack Bauer, la série a généré plusieurs niveaux de lecture, plus ou moins parodiques.

Tout porte à croire que les origines des séries télévisées vont se renouveler et se diversifier. La tendance serait à un rééquilibrage qui favoriserait l'ouverture aux différentes cultures et non une homogénéisation pétrifiante des contenus. Et, de fait, Éric Maigret et

9 Martel, p. 425 
Guillaume Soulez parlent de «fertilisations transculturelles ${ }^{10}$ » en ouverture du hors-série de MédiaMorphoses consacré aux séries télévisées (Les Raisons d'aimer les séries télé). Ces questions font débat, mais quelle que soit la position qu'on choisira d'adopter dans cette discussion, il reste important de prêter attention aux voix " émergentes ».

\section{Transpositions : intertextualité et transferts}

Les multiples mutations de la série télévisée au cours de ses pérégrinations autour du globe constituent un processus d'exportation qui apparaît comme littéral, mais qui peut aussi se voir de façon métaphorique comme la transposition du modèle constitué par l'original vers d'autres langues et d'autres contextes culturels. Il est alors possible de considérer la série comme un site de circulation culturelle : elle importe des personnages et des histoires, des références ou des citations ponctuelles, à partir de supports culturels autres (littérature, bande dessinée, etc.) ou similaires (d'autres fictions télévisées) ; elle exporte, lorsqu'elle est adaptée à destination de publics étrangers, des modèles de personnages et d'intrigues, des " formats ${ }^{11}$ ", mais aussi, parfois, sa diégèse même, lorsque son univers se déploie à travers plusieurs médias dans le cadre de la «culture de la convergence ${ }^{12}$ » et de l'hyper-sérialité.

La série est un site de relectures comme de réécritures : elle peut, en retour, venir enrichir le support auquel elle a puisé ou suggérer d'autres manières de voir et d'interpréter des œuvres connues ou canonisées. Inscrit dans les études culturelles et les théories queer, l'article de Ronan Ludot-Vlasak met ainsi au jour le fonctionnement des références intertextuelles à Keats et à Shakespeare dans la série Queer as Folk (Showtime, 2000-2005) et montre finalement comment ces allusions nous invitent à lire le canon littéraire autrement, en y décelant une subversion queer aussi latente que puissante. Au-delà d'une exploration des circulations intertextuelles, Natalie Depraz et Frédéric Mauriac proposent une étude originale de phénoménologie appliquée : ils comparent la série $D r$ House avec une véritable équipe mobile d'urgence psychiatrique, afin de mettre en lumière l'inventivité déplacée de House. La série est analysée de manière philosophique, au regard de trois facettes singulières de la mobilité : déplacement, mobilisation, créativité.

${ }^{10}$ Eric Maigret et Guillaume Soulez, « Les nouveaux territoires de la série télévisée », in Les Raisons d'aimer les séries télé, éd. Eric Maigret et Guillaume Soulez, MédiaMorphoses, Hors Série ${ }^{\circ}$ 3, 2007, p. 9 [p. 7-13].

${ }^{11}$ Albert Moran (et Justin Malbon), Understanding the Global TV Format, Bristol, Intellect Books, 2006.

${ }^{12}$ Henry Jenkins, Convergence Culture: Where Old and New Media Collide, New York et Londres, New York University Press, 2006. 


\section{La série télévisée : objet d'étude international et interdisciplinaire}

Les séries télévisées suscitent notre réflexion sur des questions qui transcendent les frontières disciplinaires, mais elles nous invitent aussi à réfléchir sur la façon dont chaque discipline peut se positionner dans le champ de recherche transdisciplinaire qui prend les séries télévisées pour objet d'étude. La série traverse les frontières entre les différentes disciplines et spécialités de recherche. Parmi les chercheurs qui s'y intéressent, certains peuvent être doublement " étrangers » à leur objet d'étude, parce qu'ils font généralement leur recherche dans une discipline qui ne prend pas traditionnellement les productions télévisuelles pour objet, et peut-être, en outre, dans une spécialité qui restreint ordinairement leur recherche à une période historique éloignée des $\mathrm{XX}^{\mathrm{e}}$ et XXI ${ }^{\mathrm{e}}$ siècles.

Des chercheurs en arts, lettres, histoire, géographie, linguistique ou philosophie, se sont, en effet, mis à étudier les séries télévisées et sont venus enrichir l'approche proposée par des disciplines qui se préoccupent presque par définition de cet objet, pour lesquelles la télévision constitue un champ de recherche dont la légitimité ne fait aucun doute. C'est le cas des sciences de l'information et de la communication et de la sociologie des médias : dans les pays francophones, on peut citer dans ce domaine d'études le hors-série de MédiaMorphoses mentionné plus haut; le numéro 165 de la revue Réseaux consacré aux séries télévisées, sorti en février 2011 et coordonné par Olivier Donnat et Dominique Pasquier ; les travaux de Jean-Pierre Esquenazi (Les Séries télévisées : l'avenir du cinéma?, Paris, Armand Colin, 2010), de François Jost (De quoi les séries américaines sont-elles le symptôme?, CNRS Éditions, 2011), de David Buxton (sociologue, auteur de Les Séries télévisées. Forme, idéologie et mode de production, L'Harmattan, 2010), de Stéphane Benassi (Séries et feuilletons TV. Pour une typologie des fictions télévisuelles, Liège, Éditions du CÉFAL, 200o), ou de Sarah Sepulchre (qui a dirigé l'ouvrage collectif Décoder les séries télévisées, Bruxelles, De Boeck, 2011).

À ces recherches, viennent donc s'ajouter celles de chercheurs en lettres, sciences humaines et études filmiques, dont on peut dater les débuts dans ce champ par plusieurs rencontres et publications : le colloque de Cerisy « Les séries télévisées », organisé par Anne Roche et Martin Winckler en août 2002 ; le livre de Barbara Villez, Séries télé. Visions de justice, publié en 2005 ; le numéro 2 de la revue GRAAT OnLine intitulé Queer Readings of Television Series and Serials et 
coordonné par Georges-Claude Guilbert et Sébastien Salbayre en juin $2007^{13}$.

Ces recherches ont pris une nouvelle impulsion depuis septembre 2009, date du colloque sur « Les pièges des nouvelles séries télévisées américaines », organisé par Sarah Hatchuel et Monica Michlin à l'université du Havre ${ }^{14}$, dans la lignée duquel s'est inscrit le colloque de Paris-Diderot, «Les séries télévisées américaines contemporaines : entre la fiction, les faits et le réel ", organisé par Ariane Hudelet et Sophie Vasset en mai 2011, colloque dont les actes constituent le premier numéro de TV/Series ${ }^{15}$. Quant au présent numéro, il rassemble une sélection d'articles tirés de communications présentées lors du colloque "Les séries télévisées dans le monde: échanges, déplacements et transpositions », que nous avons organisé à l'université du Havre, en juin de la même année.

C'est certainement dans les séries américaines récentes et le renouvellement du genre qu'elles impulsent que l'on doit chercher les raisons de l'attention portée à cet objet par les universitaires. Ces séries qui viennent concurrencer le cinéma font preuve d'une inventivité formelle et d'une réflexivité que de nombreux spécialistes d'études filmiques ou littéraires jugent pertinent d'explorer. Leur discours riche, complexe et varié sur la société américaine, l'interaction entre réalité et fiction qu'elles mettent en place, de même que le rôle de modèle qu'elles jouent dans la production télévisuelle d'autres pays, fournissent un terrain d'étude intéressant aux spécialistes de sciences humaines. Mais les capacités d'exportation, d'adaptation et de réadaptation de ces objets particuliers, à la fois œuvres fixes et programmes s'inscrivant dans un flux, peuvent aussi, sans nul doute, expliquer l'intérêt particulier qu'elles suscitent auprès de chercheurs appartenant à des domaines variés, qui dépassent le champ de l'anglistique. Le cycle de journées d'études «Philoséries » se propose ainsi, depuis 2009, de "philosopher avec les séries » telles que Buffy contre les vampires, 24 heures chrono ou encore Battlestar Galactica $^{16}$. Un numéro de la revue d'études urbaines en ligne Métropolitiques, consacré à «La ville des séries télé », explore des

\footnotetext{
${ }^{13}$ Accessible à l'adresse suivante : http://www.graat.fr/backissuequeerreadings.htm (consulté en octobre 2012).

${ }^{14}$ Voir la sélection d'articles tirés de ce colloque publiée dans le numéro 6 de GRAAT OnLine : Sarah Hatchuel et Monica Michlin (éds.), Les Pièges des nouvelles séries télévisées américaines: mécanismes narratifs et idéologiques, GRAAT On-Line, nº6, 2009 , accessible à l'adresse http://www.graat.fr/backissuepiegesseriestv.htm (consulté en octobre 2012).

15 Consultable à l'adresse: http://www.univ-lehavre.fr/ulh services/Numero-1-Issue1.html

${ }^{16}$ Voir http://philofictions.org/
} 
séries comme The Wire, Weeds ou Dexter ${ }^{17}$. Depuis 2011, la Série des séries, collection dirigée par Claire Sécail et Jean-Baptiste JeangèneVilmer aux Presses Universitaires de France, fait également appel à des chercheurs en sciences humaines pour éclairer les séries d'un regard particulier.

Le constat de l'internationalité et de l'interdisciplinarité de ce domaine de recherche soulève des interrogations, dont certaines renvoient à des problèmes de légitimité. Certains chercheurs sont-ils plus qualifiés que d'autres pour parler des séries, par exemple les spécialistes des médias ou du cinéma, ou encore les spécialistes d'une aire culturelle spécifique (coréenne, nord-américaine, sudaméricaine...)? Inversement, il peut se poser des problèmes de légitimité à l'intérieur des disciplines qui n'explorent pas traditionnellement les programmes de télévision, ou qui ont même des préoccupations implicitement en opposition à ce champ de la culture, comme les « lettres », dont le nom oriente vers le support écrit et non télévisuel, avec les jugements de valeur non-dits que ceci a tendance à accompagner. S'ajoute le fait que les auteurs de séries semblent euxmêmes avoir une certaine réticence à considérer leurs œuvres comme comparables à de la littérature ${ }^{18}$. Devrait-on, dès lors, annexer l'étude des séries télévisées à une discipline plus reconnue, et bénéficier ainsi de sa légitimité ? Devrait-il exister une discipline « séries télévisées »? Parce que les échanges entre les disciplines n'excluent en aucun cas débats et discussions, et apparaissent comme éminemment productifs, créer une nouvelle discipline « autonome » serait, à nos yeux, se priver de perspectives, de méthodologies, d'angles d'approche spécifiques aux diverses disciplines à même de produire une recherche inédite, riche, variée et évolutive.

\section{Bibliographie}

Benassi Stéphane, Séries et feuilletons TV. Pour une typologie des fictions télévisuelles, Liège, Éditions du CÉFAL, 2000

BEuve-MÉRY Alain, "La série télé, ce n'est pas de la littérature », Le Monde.fr, le 29 mai 2011,

http://www.lemonde.fr/culture/article/2011/05/29/la-serie-tele-cen-est-pas-de-la-litterature_1529071_3246.html

${ }_{17}$ Philippe Simay et Stéphane Tonnelat (éds), « La ville des séries télé », Métropolitiques, 7 novembre 2011, http://www.metropolitiques.eu/La-ville-des-series-tele.html (consulté en novembre 2012)

${ }^{18}$ Voir par exemple les propos d'Eric Overmyer, créateur avec David Simon de Treme (HBO, 2010-), dans Alain Beuve-Méry, "La série télé, ce n'est pas de la littérature », Le Monde.fr, le 29 mai 2011, http://www.lemonde.fr/culture/article/2011/05/29/la-serietele-ce-n-est-pas-de-la-litterature_1529071_3246.html (consulté en novembre 2012). 
Buxton David, Les Séries télévisées. Forme, idéologie et mode de production, L'Harmattan, 2010

DonNat Olivier et Dominique PASQuier (éds), Les Séries télévisées, Réseaux, $\mathrm{n}^{\circ}$ 165, 2011.

EsquenaZI Jean-Pierre, Les Séries télévisées : l'avenir du cinéma ?, Paris, Armand Colin, 2010.

GILLESPIE Nick et Matt WELCH, « How Dallas Won the Cold War », The Washington Post, 27 avril 2008, http://www.washingtonpost.com/wpdyn/content/article/2008/04/25/AR2008042503103.html

GuILBERT Georges-Claude et Sébastien SALBAYRE (éds.), Queer Readings of Television Series and Serials, GRAAT On-Line, ${ }^{\circ} 2,2007$, http://www.graat.fr/backissuequeerreadings.htm

Hatchuel Sarah et Monica Michlin (éds.), Les Pièges des nouvelles séries télévisées américaines: mécanismes narratifs et idéologiques, GRAAT On-Line, $\mathrm{n}^{\circ} 6,2009$,

http://www.graat.fr/backissuepiegesseriestv.htm

HudELET Ariane et Sophie VASSET (éds.), Les Séries télévisées américaines contemporaines : Entre la fiction, les faits, et le réel / Contemporary American TV Series: Between Fiction, Fact, and the Real, TV/Series, $\mathrm{n}^{\circ}$ 1, 2012,

http://www.univ-lehavre.fr/ulh_services/Numero-1-Issue-1.html

JEnkIns Henry, Convergence Culture: Where Old and New Media Collide, New York et Londres, New York University Press, 2006.

Jost François De quoi les séries américaines sont-elles le symptôme?, CNRS Éditions, 2011.

«La 'telenovela' se réinvente pour le public latino aux États-Unis », L'Express.fr, 31 août 2011,

http://www.lexpress.fr/actualites/1/economie/la-telenovela-sereinvente-pour-le-public-latino-aux-etats-unis_1025732.htm

MAIGRET Éric et Guillaume Soulez (éds.), Les Raisons d'aimer les séries télé, MédiaMorphoses, Hors Série nº 3, 2007.

MARTEL Frédéric, Mainstream, Enquête sur cette culture qui plaît à tout le monde, Flammarion, 2010. 
Moran Albert et Justin MaLBon, Understanding the Global TV Format, Bristol, Intellect Books, 2006.

Mоттет Jean, «L'espace-temps de la télévision : le cas du soap opera », Quaderni, n ${ }^{\circ}$, 1989-1990, p. 65-78.

NYE Joseph, Bound to Lead: The Changing Nature of American Power, New York, Basic Books, 1990.

Soft Power: The Means to Success in World Politics, New York, Public Affairs, 2004

SePulchre Sarah (éd.), Décoder les séries télévisées, Bruxelles, De Boeck, 2011.

Simay Philippe et Stéphane TonNELAT (éds), « La ville des séries télé », Métropolitiques, 2011, http://www.metropolitiques.eu/La-ville-des$\underline{\text { series-tele.html }}$

VILlez Barbara, Séries télé. Visions de justice, Paris, Presses Universitaires de France, 2005. 